\title{
Stimulus information and stimulus interference in bisensory short-term memory'
}

\author{
RHEA L. DORNBUSH ${ }^{2}$ \\ NEW YORK MEDICAL COLLEGE, FLOWER AND FIFTH AVENUE HOSPITALS
}

\begin{abstract}
An attempt was made to determine if Ss' strategy of retaining stimulus material changed under conditions of bisensory simultaneous stimulation when one of the two inputs was relevant information as opposed to interference. Thus in Group 1 Ss were stimulated bisensorily, attended to both inputs (information), but recalled only one input. Ss in Group 2 were stimulated bisensorily, attended to only one input, and recalled only that input (interference). It was found that $S$ 's efficiency of recall was decreased in the information condition (Group 1) but strategy was not altered. In both groups, Ss performed similarly; performance was in turn similar to that observed in the typical bisensory situation, i.e., where both channels are recalled.
\end{abstract}

In experiments concerned with different but simultaneous inputs such as in the dichotic or bisensory presentation of material, the question is immediately raised as to whether $S$ can, in fact, effectively handle two inputs and whether strategy of encoding and storing, etc., changes under such conditions of presentation.

Apparently, under conditions of dichotic stimulation, strategy does change. More specifically, in simple auditory memory span experiments, fast presentation of material is superior to slow presentation, i.e., a decay function is obtained. However, with two different auditory inputs more efficient recall is usually found with slow rather than fast presentation rates (Broadbent, 1954; Moray, 1960). Broadbent and Gregory (1961) suggested that "It is only when digits are arriving by two separate sensory channels that slow presentation is superior to fast. In simple memory spans the exact opposite is true.... Thus the better performance at slow presentation rates ... must be connected with the use of two channels [Broadbent \& Gregory, 1961, p. 107]."

Broadbent (1954) found that Ss attending to different sets of digits presented simultaneously to both ears, as in: Right, 723; Left, 615, generally attempted to recall all of the material from one ear first and then from the other ear (e.g., 723516 or 516723 ). With a slow delivery rate (one pair of digits $/ 2 \mathrm{sec}$ ), however, recall was possible in the order of arrival of the digits, i.e., pair by pair (e.g., 752136). Broadbent reasoned that the slower rate allowed for switching from one ear to the other for each pair of digits; at the faster rates $S$ was not able to switch from ear to ear fast enough. Thus he adopted the strategy of attending to all of the information from one channel first and then the other.

On the other hand, in bisensory situations employing the auditory and visual modalities, S's efficiency of recall is lessened; however, his strategy of handling the simultaneous inputs does not change. That is, recall in each modality is similar to recall when each is stimulated separately (Dornbush, 1968); a decay function is obtained in the auditory modality while visual recall increases with decreasing or slowing rate (Mackworth, 1962).

Thus, in the bisensory situation it would seem that altering S's task and at the same time the function of the second input, would change only efficiency of recall, whereas the pattern of recall would remain the same. Therefore, the present experiment sought to determine the effects in efficiency and pattern of recall of the second input when it was in one situation relevant information and in a second situation merely interference. To this end Ss were stimulated bisensorily and required to attend to both inputs but recall only one; here the second input was information, as $\mathrm{S}$ did not know until after material presentation which set he could discard. A second group of Ss was stimulated bisensorily, attended to only one modality and recalled that modality; the unattended input served as interference.

\section{Apparatus}

\section{METHOD}

Materials were presented on 16-mm film (24 frames/sec) with synchronous sound track by means of a Kalart projector. The exact auditory sequences intended for pairing with the appropriate visual sequences were recorded in a sound studio by the E; precise control of synchrony was accomplished by splicing the auditory tape. That is, speech recordings were spliced into the film so that each utterance of a stimulus item commenced within the first half frame of its associated visual stimulus $( \pm 21 \mathrm{msec})$.

Visual stimuli were made by Letraset instant black letters exposed singly on white cards, $81 / 2 \times 11$ in., which were then filmed and appropriately edited. The contrast ratio as displayed was approximately $80 \%$. The interstimulus and interlist intervals for visually presented materials consisted of clear leader which was slightly less bright than the stimulus sequence, thus minimizing the negative afterimage. While an attempt was made to subjectively equate the auditory and visual intensities it seemed reasonable to assume that the intensities might not be the crucial variable so long as the material was clearly and easily discriminable.

\section{List Preparation and Presentation}

The first 10 letters of the alphabet, A through $\mathrm{J}$, and the digits 0 through 9 , were employed. As materials were not the main concern and had been investigated previously (Dornbush, $1968 \mathrm{a}, \mathrm{b})$ data was not analyzed with respect to material combinations.

Altogether, 24 pairs of lists, each list containing six items, were required. For half of the lists $S s$ were required to report just the visual modality, and for the remaining half just the auditory modality.

Visual items were presented for $.5 \mathrm{sec}$ ( 12 frames); the durations of the auditory items averaged 10 frames or $.042 \mathrm{sec}$. Materials were presented at three rates: (1) one pair $/ .5 \mathrm{sec}$, no interstimulus interval (fast); (2) one pair/sec, .5 -sec interstimulus interval (medium); (3) one pair $/ 2 \mathrm{sec}$, $1.5-\mathrm{sec}$ interstimulus interval (slow). There was a $20-\mathrm{sec}$ interlist interval, with $15 \mathrm{sec}$ for recall and a ready signal given $.5 \mathrm{sec}$ before the start of each list.

All lists at one rate were presented before the lists at the other rates, in the medium, slow, fast order.

\section{Subjects}

One hundred and twenty undergraduate psychology students satisfying a course requirement served as Ss. Sixty were in Group 1 and 60 in Group 2.

\section{Administration \\ Subjects were run as a group in a large auditorium with projection booth. They were centrally seated so that no $S$}


Table 1

Means and (SDs) for the Main Effects of Group, Modality, and Rate

\begin{tabular}{lrrrrr}
\hline Group Infor: & $3.27(1.14)$ & Inter: & $5.02(.98)$ & \\
Modality Aud: & $4.50(1.22)$ & Vis: & $3.80(1.40)$ & \\
Rate $\quad$ F: & $4.19(1.34)$ & M: & $4.07(1.36)$ & S: $4.19(1.37)$ \\
\hline
\end{tabular}

viewed the screen at an extreme angle. All Ss were given general short-term memory instructions with emphasis placed on the necessity of correct serial order of items in written response. In Group 1, immediately after the presentation of the last item in each list, the word "auditory" or "visual" was spoken indicating which channel to recall; in Group 2, the word "auditory" or "visual," again indicating channel to be recalled, occurred before the ready signal. Thus, Group I was the information group and Group 2 the interference group. Channel-to-be-recalled was determined by prearranged random order.

\section{RESULTS AND DISCUSSION}

The data for each $S$ for each sequence of six items were scored according to the accuracy of responses both in nature and location, i.e., on an item-by-item basis permitting individual scores to take on values between 0 and 6 . The means and standard deviations for each of the main effects are shown in Table 1 and the results of the analysis of variance are shown in Table 2.

The analysis of variance indicates that the main effects of Group and Modality were both highly significant $(p<.001)$. The direction of the difference in Groups is as was expected: More was recalled in the situation where $S$ had to attend to one input (Group 2) rather than two (Group 1). For Modality, auditory recall was greater than visual recall-results which are typically obtained (Broadbent, 1958; Buschke, 1962; Dornbush, 1968a, b; Margrain, 1967).

Additionally, the interactions of Modality by Rate and Groups by Rate were significant $(p<.001)$. In the first interaction, recall was best in the visual modality at the

Table 2

Summary of the Analysis of Variance for the Group by Modality by Rate Interaction

\begin{tabular}{lrrrrc}
\hline Source & \multicolumn{1}{c}{ SS } & DF & MS & F & P \\
\hline Between Ss & 829.82 & 119 & & & \\
A (Group) & 546.89 & 1 & 546.89 & 227.81 & $<.001$ \\
Ss WG & 282.93 & 118 & 2.40 & & \\
Within Ss & 510.03 & 600 & & & \\
B (Modality) & 88.90 & 1 & 88.90 & 107.11 & $<.001$ \\
AB & .90 & 1 & .90 & 1.08 & - \\
B x Ss WG & 98.27 & 118 & .83 &.. & \\
C (Rate) & 2.36 & 2 & 1.18 & 2.14 & - \\
AC & 7.81 & 2 & 3.90 & 7.09 & $<.001$ \\
C x Ss WG & 129.24 & 236 & .55 & & \\
BC & 30.52 & 2 & 15.26 & 23.80 & $<.001$ \\
ABC & 1.67 & 2 & .84 & 1.31 & - \\
BC x Ss WG & 150.36 & 236 & .64 & & \\
\hline
\end{tabular}

slowest rate, and in the auditory modality, at the fastest rate. The nature of this interaction accounts for the weak discrimination attributed to rate as a main effect (Table 1).

In the second interaction, when the second channel is information, recall is best at the fastest rate; when the second channel is interference, recall is best at the slowest rate. The direction of this interaction was determined, not by any changes in strategy, but by larger differences in recall, in Group 1, at each rate within the auditory modality thus imposing on this group auditory rate characteristics; similarly, there were larger differences in recall, in Group 2, at each rate within the visual modality thus imposing on this group visual rate characteristics.

Most important, however, in light of the present concern, was the nonsignificant Group by Modality by Rate interaction. This data confirms the suggestion that strategy does not change in bisensory situations, but efficiency does. In both groups a decay function was obtained in the auditory modality whereas an increasing function was obtained with visual material.

Thus, overall recall was better when $\mathrm{S}$ did not have to retain two sets of materials even though only one set was recalled. However, the relationships among variables which are typically obtained in unisensory and bisensory situations remain the same regardless of whether one input acts as information or as interference. That is, $\mathbf{S}$ does not significantly alter strategy of retaining stimulus information when confronted with multiple inputs.

\section{REFERENCES}

BROADBENT, D. E. The role of auditory localization in attention and memory span. Journal of Experimental Psychology, 1954, 47, 191-196.

BROADBENT, D. E. Perception and communication. London: Pergamon Press, 1958.

BROADBENT, D. E., \& GREGORY, M. On the recall of stimuli presented alternately to two sense-organs. Quarterly Journal of Experimental Psychology, 1961, 13, 103-109.

BUSCHKE, H. Auditory and visual interaction in immediate memory. Journal of Psychiatric Research, 1962, 1, 229-237.

DORNBUSH, R. L. Input variables in bisensory memory. Perception \& Psychophysics, 1968a, 4, 41-44.

DORNBUSH, R. L. Shadowing in bisensory memory. Quarterly Joumal of Experimental Psychology, 1968b, 20, 225-231.

MACKWORTH, J. F. Presentation rate and immediate memory. Canadian Journal of Psychology, 1962, 16, 43-47.

MARGRAIN, S. A. Short term memory as a function of input modality. Quarterly Journal of Experimental Psychology, 1967, 19, 109-114.

MORAY, N. Broadbent's filter theory: Postulate $\mathrm{H}$ and the problem of switching time. Quarterly Journal of Experimental Psychology, 1960, $12,214-220$.

\section{NOTES}

1. Supported by grants from USPHS (MH 15600), the American Philosophical Society, and The Society of the Sigma Xi

2. Address: Department of Psychiatry, New York Medical College, Flower and Fifth Avenue Hospitals, Five East 102nd Street, New York, New York 10029.

(Accepted for publication December 13, 1968.) 